Abstracted/indexed in Academic Search Complete, Asia Journals Online, Bangladesh Journals Online, Biological Abstracts, BIOSIS Previews, CAB

Abstracts, Current Abstracts, Directory of Open Access Journals, EMBASE/Excerpta Medica, Google Scholar, HINARI (WHO), International

\title{
Neuroprotective effects of electroacupuncture on hypoxic-ischemic encephalopathy in newborn rats association with increased expression of $\mathrm{mTOR}$
}

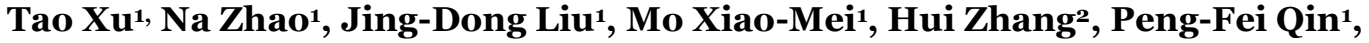 \\ Xiang-Chun Feng' and Qing Sun ${ }^{1}$
}

${ }^{1}$ Qingdao Women and Children Hospital, Qingdao, Shangdong, 266 000, P.R. China; ${ }^{2}$ Qingdao University Medical College, Qingdao, Shangdong, 266 000, P.R. China.



\begin{abstract}
In this study, we observed the therapeutic effects of acupuncture and investigated the underlying molecular mechanisms by constructed a hypoxicischemic encephalopathy (HIE) animal model. In the electroacupuncture group, mTOR expression increased since $1 \mathrm{~d}$, and continued to rise till the $21^{\text {st }}$ day. All of the differences were significantly ( $p<0.05$ vs the model group). Meanwhile, mTOR expression was analyzed by Western blotting. There was statistical significance between the model group and the electroacupuncture group in the four time periods $(\mathrm{p}<0.05)$. The results provide evidence that electroacupuncture treatment protected cortical neurons against HIE-induced neuronal damage and degenerative changes in rats, which is in association with activation of mTOR both at the mRNA level and protein level. Therefore, electroacupuncture may become a potential therapeutic strategy for HIE of newborn.
\end{abstract}

\section{Introduction}

Hypoxic-ischemic encephalopathy (HIE) is an important cause of brain injury in the newborn and can result in long-term devastating consequences. There has been amounting research progress in HIE over the last two decades and many target molecules have been found. Despite all these advances, therapeutic interventions are still limited (Lai and Yang, 2011; Fatemi et al., 2009).

After neonatal hypoxic-ischemic disease happening, there was a significant increase in neural autophagy in a short time (Koike et al., 2008; Rudov et al., 2014; Bellot et al., 2009). Autophagy is reported to play a predominant role in the pathological progress of hypoxia-ischemia brain damage ( $\mathrm{Li}$ et al., 2007). Previous studies found that the phosphatidylinositol 3kinase/protein kinase B (PI3K/AKT) signaling pathway was involved in the survival process after hypoxia -ischemia brain damage through regulating the expression of some relative protein (Fahling, 2009). PI3-K/ Akt is an important pathway related to cell fate determination.

The mammalian target of rapamycin (mTOR), an atypical Ser/Thr protein kinase, is a main down-stream of PI3K/AKT signaling pathway (Nakamura et al., 2006; Gunn and Hailes, 2008; Kang et al., 2008). Previous study found that mTOR as a key regulator of autophagy, could regulate cell growth, cell apoptosis, energy metabolism and inhibition of autophagy after activation. It plays as a role in brain protection. When nutritional status, growth circumstance, hormone levels, and other conditions changes, mTOR has special residues phosphorylation, and changes its activity. Thus, it can play an important role in the processes of many diseases (Mazure and Pouysségur, 2010; Blagosklonny, 2013).

As an alternative medicine methodology originating in 
ancient China, acupuncture has been used for more than 1,000 years as a treatment or as an adjuvant modality for patients with stroke. It has been frequently used in Asian countries and has become increasingly popular in the western world (MacPherson and Hammerschlag, 2012; Fang et al., 2012; Ji and Zhang, 2009; Zhou et al., 2009).

Several animal studies have indicated neuroprotective effect of acupunture on hypoxic-ischemic injury. It was shown to play an important role in functional reorganization and cerebral compensation (Wang et al., 2005; Yi et al, 2006; Yang et al., 2005; Li et al., 2012; Gao et al., 2011). The possible mechanisms involved have attracted more and more attentions. So, using a HIE rat model, in the present study we evaluated the in vivo therapeutic efficacy of electroacupuncture and investigated the underlying molecular mechanisms, especially the role of mTOR in the process.

\section{Materials and Methods}

This study was performed at the Experimental Center, Medical College of Qingdao University Affiliated Hospital, from September 2013 to May 2014.

\section{Animal}

A total of 236 seven-day-old SD rats (belonging to 11 broods with the mothers included, 10 to 11 newborn rats per brood), male or female, breast-feeding, specific pathogen-free (SPF) grade, weighing 10.5 to $15 \mathrm{~g}$ (average weight, $112.8 \pm 1.6 \mathrm{~g}$ ), were provided by Qingdao Drug Detection Institute (No. SCXK (Lu) 20130001). They were maintained in a temperaturecontrolled $\left(20\right.$ to $\left.25^{\circ} \mathrm{C}\right)$ facility with a 12 hours light/12 hours dark cycle. Among which four weighing less than $11 \mathrm{~g}$ were excluded. In each brood, it is randomly assigned into two groups, sham-surgery group and HIE model group. Totally there were 25 rats in the former group and 211 rats in the latter group.

Finally 176 rats were successfully established as the HIE model with the successful rate of $83.4 \%$. Then the successfully established rats were randomly divided into the HIE model group, sham-acpuncture group, acupuncture group, antagonists group, and acupuncture and antagonists group, with 35 rats in each group. All groups were subdivided into four subgroups in terms of four time periods of $1 d, 3 d, 7 d$ and $21 d$.

\section{Establishment of HIE animal model}

The HIE model was constructed according to the LCCA ligation method in combination with hypoxic treatment described by Rice et al. (1981). Briefly, animals were anesthetized by inhaling ether. In the supine position, a midline ventral incision was made to expose the LCCA, which was carefully separated from the vagus nerve. The LCCA was separated, ligated using a 5/0 silk suture, and then the incision was sewed up. After a two -hour restoration, the rat was put in a sealed transparent vessel and set in the warm bathe with the temperature of $37^{\circ} \mathrm{C}$. Then the vessel was passed into gas with low content of oxygen (including $8 \%$ of oxygen and $92 \%$ of nitrogen) at the velocity of $1 \mathrm{~L} / \mathrm{min}$ for 2.5 hours. The survivors were kept warm for another one hour and then received behavioral tests. For the sham-surgery group, after anesthesia, the LCCA was separated without ligation and the incision was sewed up without any hypoxic treatment. The rat died or not awake 2 hours after surgery were excluded. Behavioral tests were made four hours later, and rats in this group showed no behavioral abnormality.

\section{Electroacupuncture treatment}

The bilateral common carotid arteries were separated, without ligation, and the rats were without hypoxia after incisions sutured in the sham surgery group. The bilateral common carotid arteries were separated and ligated, and incisions were sutured, without any other treatment in model group.

In the electroacupuncture group, four acupoints including Baihui (GV20), Dazhui (GV14), Quchi (LI11) and Yongquan (KI1) were chosen, positioned according to the ordinary acupoints for acupuncture in rats. Localization of the acupoints was based on the International Standard Scheme for acupoint names of acupuncture drafted by the experimental acupuncture branch of the China Acupuncture Academy. Baihui (GV20) acupoint is located in the center of the parietal bone. Dazhui (GV14) is located between the seventh cervical vertebra and the first thoracic vertebra, just in the center of the back. Quchi (LI11) is in the midpoint of the line between the outer end of the elbow stripes and the epicondyle of the humerus, and Yongquan (KI1) is located in the plantar anterior third (toes excluded). Needles 0.5 inch in length were horizontally inserted backwards into Baihui (GV20) for 0.2 inch deep, perpendicularly inserted into Dazhui (GV14) for 0.2 inch deep, perpendicularly inserted into Quchi (LI11) for 0.5 inch deep and rapidly inserted into Yongquan (KI1) without leaving the needle, respectively. The two acupoints Baihui (GV20) and Quchi (LI11) were connected with G-6805 electric acupuncture apparatus (Shanghai Huayi Medical Instrument Factory, China), and receive electroacupuncture for $10 \mathrm{~min}$ with the continuous wave at the frequency of 5 to $10 \mathrm{~Hz}$ with the local tissue shivering slightly, and with the voltage between 3 to $5 \mathrm{~V}$. The acupuncture therapy was given once a day for consecutive day 1, 3, 7 and 21 respectively. The positions were from Baihui (GV20), Dazhui (GV14), Quchi (LI11) and Yongquan (KI1) by 1 $\mathrm{cm}$. In the antagonists group the HIE model rats were injected wortmannin $5 \mathrm{uL}(20 \mathrm{ug} / \mathrm{kg}$ wortmannin, dissolved in DMSO) to the left ventricle by stereo positioning instrument. The acupuncture and anta- 
gonists group had the operations of antagonists group and electroacupuncture group at the same time. Hematoxylin-eosin (HE) staining for determination of
neuronal damage

Rats were anesthetized by inhaling ether at the indicated time. The left ventricle was cannulated and perfused with phosphate-buffered saline (PBS) (preheated at $37^{\circ} \mathrm{C}$ ), and then perfused and fixed with $4 \%(\mathrm{w} / \mathrm{v}$ ) paraformaldehyde (in 0.1 M PBS ( $\mathrm{pH} 7.4$ ), precooled at $4^{\circ} \mathrm{C}$ ) firstly at full speed till convulsion of the limbs ceased, then perfusion was kept at the velocity of $1 \mathrm{~mL} / \mathrm{min}$. Then, the brain was quickly separated with the cerebellum and the brainstem removed and placed in $4 \%$ paraformaldehyde for 24 hours at $4^{\circ} \mathrm{C}$. The specimens were dehydrated with $20 \%$ sucrose and frozen. The frozen sections were then serially cut into $20 \mu \mathrm{m}$ thick coronal slices. HE staining was performed according to the standard protocol The sections were observed under a light microscope (Nikon, Japan) using a magnification of $\times 200$, and photographed.

\section{Detection of RET mRNA expression in the injured cortex by real time RT-PCR}

Total RNA was extracted from the injured cortex (about $1 \mathrm{~mm} \times 1 \mathrm{~mm} \times 1 \mathrm{~mm}$ ) of rats using Trizol (Invitrogen, USA) according to the manufacturer's instructions. Two micrograms of total RNA were used to synthesize firststrand cDNA with M-MuLV reverse transcriptase (Fermentas, USA) using random primers. Real time PCR was performed using the ABI 7500 real time PCR detection system (ABI, USA) with SYBR green (Fermentas, USA). Primer sequences for specific genes are presented as follows. Forward primer for mTOR: $5^{\prime}$ CGCTGATCCCTTTATCG-3' ; Reverse primer for mTOR: 5'-ATGCTCAAACACCTCCACC-3'(168bp). Forward primer for GAPDH: 5'-GAAGGTGAAGGTCGGAGT-3'; Reverse primer for GAPDH: 5'-GAAGATGGTGATGGGATTTC-3' (193bp). GAPDH was used as an internal control. Detection of $m T O R$ protein expression in the injured
cortex by western blot analysis

Total protein extracts were prepared from rat brains as previously described. In brief, rats were anesthetized with ether and rapidly decapitated. Brain was quickly separated with the cerebellum and the brainstem removed and placed on ice in 10 volumes of cold homogenization buffer $(50 \mathrm{mmol} / \mathrm{L}$ Tris, $120 \mathrm{mmol} / \mathrm{L}$ $\mathrm{NaCl}, \mathrm{pH}$ 7.4) to which protease inhibitors (Sigma, USA) had been added. The tissue was then homogenized and stored at $-80^{\circ} \mathrm{C}$. Protein concentrations were determined using the Bradford method (Bio-Rad, USA). Equal amounts of protein $(50 \mu \mathrm{g} / \mathrm{lane})$ were separated by SDS-PAGE and transferred onto polyvinylidene difluoride membranes (Millipore, USA). After being blocked, the filters were incubated with the following primary antibodies: mTOR monoclonal antibody (Cell Signaling, USA). Anti-GAPDH antibody (Abcam, USA). GAPDH was used as an internal loading control. After being washed and incubated with the goat anti rabbit secondary antibody (Maixin Company, China), the immune complexes were visualized with a chemiluminescence reagent. Western blots were quantified densitometrically with Quantity One software (Bio-Rad, USA), and the intensity values were normalized to GAPDH.

\section{Statistical analysis}

All data were analyzed using SPSS 13.0 software (SPSS, Chicago, IL, USA). Measurement data were expressed as mean $\pm \mathrm{SD}$. Comparisons among several groups were made using one-way analysis of variance under equal variances and Kruskal-wallis $\mathrm{H}$ analysis under unequal variances. Comparisons between groups were made using SNK-q test under equal variances and Dunnett's T3 test under unequal variances. $p<0.05$ (two -tail) was considered statistically significant.

\section{Results}

Electroacupuncture treatment significantly ameliorates neuronal damage caused by hypoxic-ischemic injury

In the sham-surgery group, HE staining showed normal structures of cortex with clear organizational structure, normal cell outline, clear nucleolus and cell nucleus located in the center (Figure 1- F). In the control model group, hypoxic-ischemic injury for $1 \mathrm{~d}$ to $3 \mathrm{~d}$ induced neuronal damage with obvious signs of necrosis and degeneration of neurons, such as mesh-like structure, irregular arrangements of neurons, concentrated cytoplasm, vacuolation of cytoplasm, karyopyknosis, and nucleolus loss (Figure 1-A1, A3). 7d to 21d after the operation, severe neuron loss along with proliferation of surrounding astrocytes was observed (Figure 1-A7, A21). The pathological changes of nerve cells in the sham acupuncture group (Figure 1-B1, B3, B7, B21) were similar to that in the model group. Electroacupuncture for $3 \mathrm{~d}$ showed swelling and degeneration of neurons but less severe than the control model group (Figure 1-C3). Electroacupuncture treatment for $7 \mathrm{~d}$ to 21d showed less signs of necrosis and degeneration of neurons, that is relatively clear organizational structure, regular arrangements of neurons, normal cell outline and relatively clear nucleolus (Figure 1-C7, C21). In acupuncture and antagonists group, pathological changes of nerve cells also had a trend to reduce, but inferior to the acupuncture group (Figure 1-E3, E7, E21). In antagonists group, more obvious nerve cell necrosis, cell shrinkage, nuclear condensations were seen (Figure 1-D1, D3, D7, D21).

Expression changes of mTOR mRNA in the cerebral cortex of rats from different time sessions of experimental groups 


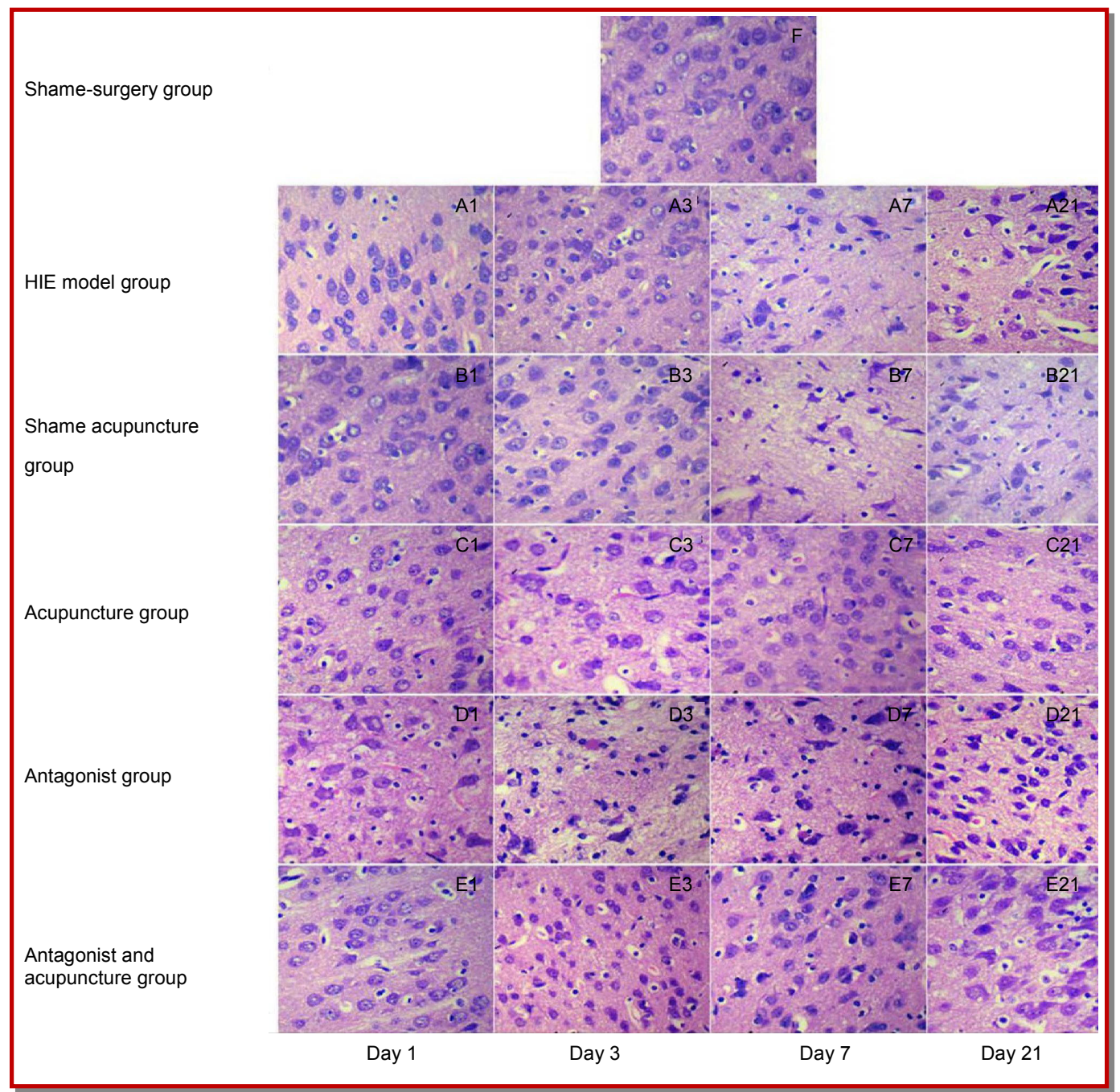

Figure 1: Effect of electroacupuncture treatment on neuronal damage in the injured cortex of rat with HIE (Hematoxylin-eosin staining, optical microscope, $\times 200)$.

MTOR mRNA expression levels were detected with realtime RT-PCR. As was shown in Figure 2, compared with that of the sham-surgery group, expression levels of mTOR mRNA in the cerebral cortex with hypoxicischemic injury from the model group increased at the first day post-surgery $(p<0.05)$, continued increasing three days later and reached the peak at the seventh day $(p<0.05)$. To 21 days, the expression of mTOR mRNA in model group was not significantly increased, but compared with the sham operation group, and the difference was also significantly $(p<0.05)$. The expression in the sham-acupuncture group was close to that of the model group with sustained rising in $1 \mathrm{~d}, 3 \mathrm{~d}$, $7 \mathrm{~d}$ and $21 \mathrm{~d}$, and the differences were not significant $(p>0.05)$. In the electroacupuncture group, mTOR expression also increased since the first day, and continued to rise till the $21^{\text {st }}$ day. All of the differences were significantly ( $<<0.05$ vs the sham-surgery group). The expressions of mTOR in the acupuncture and antagonists group were significantly different from that of the sham-surgery group in $1 \mathrm{~d}, 3 \mathrm{~d}$ and $7 \mathrm{~d}$, and to $21 \mathrm{~d}$, the differences were significant compared to that of model group $(p<0.05)$. Table I showed the changes of mTOR mRNA in each group at different times.

Expression changes of mTOR protein in the cerebral cortex of rats from different time sessions and different groups

MTOR expression was analyzed by western blotting. In comparison with the sham-surgery group, mTOR expression at the protein level was significantly increased both in model group $(\mathrm{p}<0.05)$ and in the electroacupuncture group $(\mathrm{p}<0.05)$ at the $1 \mathrm{st}, 3 \mathrm{rd}, 7 \mathrm{th}$, 21 th day. Furthermore, mTOR expression was increased both in the model group and in electroacupuncture group. There was statistical significance between the model group and the electroacupuncture group in the four time periods $(\mathrm{p}<0.05)$. MTOR expressions in electroacupuncture and antagonists group were not significant compared to model group at 1st, 3rd, 7 th day $(\mathrm{p}>0.05)$, but the difference was significant at $21 \mathrm{rd}$ day $(\mathrm{p}<0.05)$ (Figure 3$)$. 


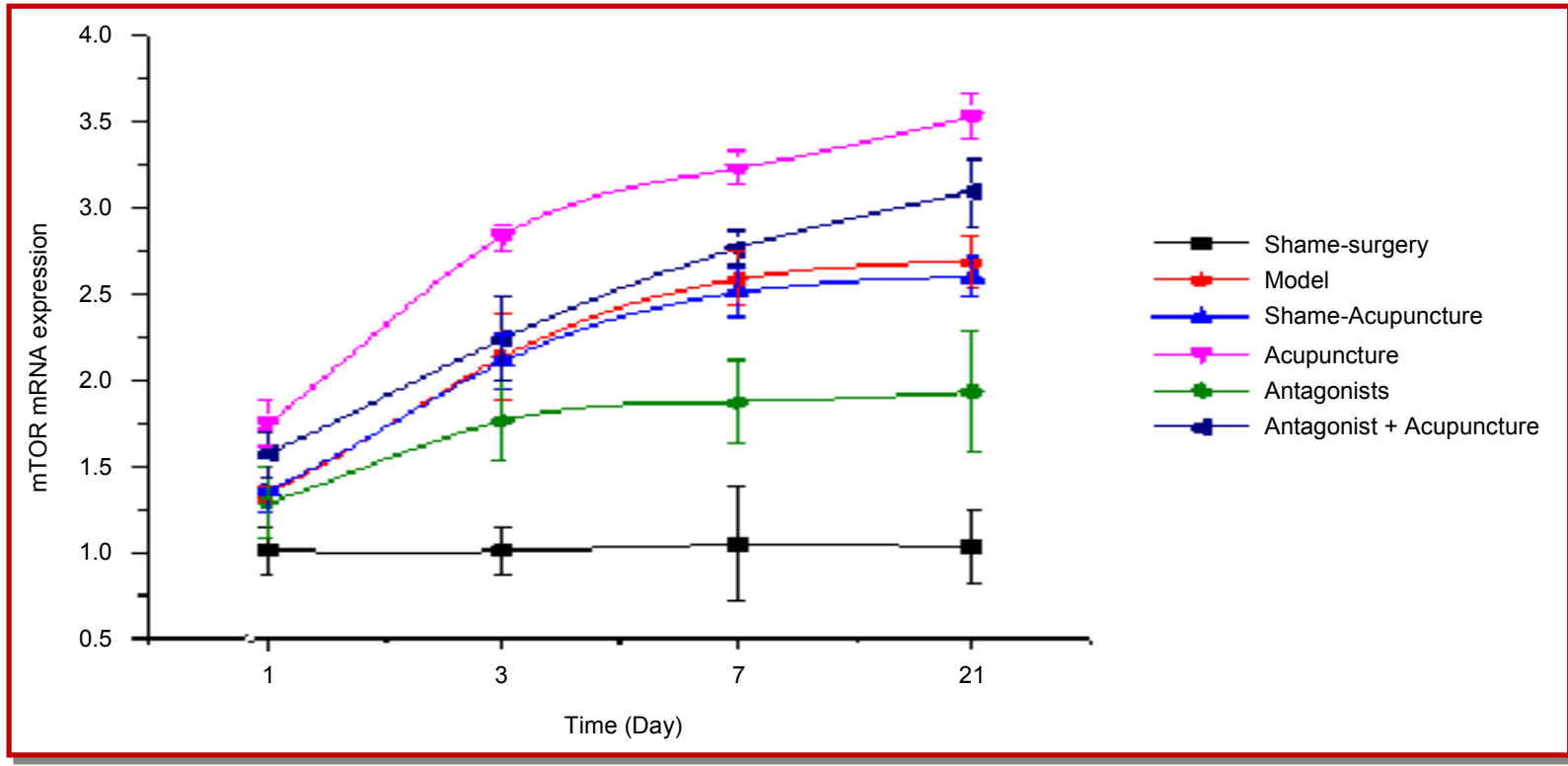

Figure 2: Effect of electroacupuncture treatment on mTOR mRNA expression in the injured cortex of rat with HIE (real time RTPCR)

\begin{tabular}{|lcccc|}
\hline \multicolumn{5}{|c|}{ Table I } \\
\hline \multicolumn{5}{|c|}{ The expression of mTOR mRNA in hypoxic ischemic brain tissue } \\
\hline Group & Day 1 & Day 3 & Day 7 & Day 21 \\
\hline Sham-surgery & $1.0 \pm 0.1$ & $1.0 \pm 0.1$ & $1.0 \pm 0.3379$ & $1.0 \pm 0.2$ \\
Model & $1.3 \pm 0.0^{\mathrm{a}}$ & $2.1 \pm 0.2^{\mathrm{a}}$ & $2.6 \pm 0.2^{\mathrm{a}}$ & $2.7 \pm 0.1^{\mathrm{a}}$ \\
Sham-acupuncture & $1.4 \pm 0.1^{\mathrm{a}}$ & $2.1 \pm 0.2^{\mathrm{a}}$ & $2.5 \pm 0.1^{\mathrm{a}}$ & $2.6 \pm 0.1^{\mathrm{a}}$ \\
Acupuncture & $1.8 \pm 0.1^{\mathrm{abc}}$ & $2.8 \pm 0.1^{\mathrm{abc}}$ & $3.2 \pm 0.1^{\mathrm{abc}}$ & $3.5 \pm 0.1^{\mathrm{abc}}$ \\
Antagonists & $1.3 \pm 0.2$ & $1.8 \pm 0.2$ & $1.9 \pm 0.2$ & $1.9 \pm 0.4$ \\
Acupuncture and antagonists & $1.6 \pm 0.1^{\mathrm{a}}$ & $2.2 \pm 0.2^{\mathrm{a}}$ & $2.8 \pm 0.1^{\mathrm{a}}$ & $3.1 \pm 0.2^{\mathrm{abc}}$ \\
\hline
\end{tabular}

mRNA was normalized to the internal control GAPDH. Data are expressed as mean \pm SD; $n=6$ rats per group; ap $<0.05$ vs the corresponding sham-surgery group; ${ }^{b} p<0.05$ vs the corresponding model group; $c p<0.05$ vs the corresponding antagonists group)

\section{Discussion}

In the present study, we constructed a HIE animal model to evaluate the in vivo therapeutic efficacy of electroacupuncture and to further investigate the role of mTOR in the process. Cerebral development of sevenday-old rat is similar to that of the newborn, so we chose seven-day-old rat to construct the model. Internal carotid artery and vertebral artery of rats form Willis ring at the undersurface of cerebrum. Therefore ligation of one side of the common carotid arteries cannot induce ideal ischemic injury. In 1981, Rice firstly established a rat model of HIE by ligating the common carotid artery in combination with hypoxic treatment (Rice et al., 1981). Since then, the model has been widely used for its simple operation, high successful rate, low mortality, high reliability and good reproducibility.

Many animal studies have indicated neuroprotective effect of acupunture on hypoxic-ischemic injury. Acupoint combination is a complicated issue. Among different acupoints, Baihui (GV20) and Dazhui (GV14) are the main points of the Du channel, which exhibits a combined supervising effect on the entire meridian system. Acupuncture at Baihui in combination with Dazhui promoted flow of blood, resuscitated consciousness, recuperated depleted yang, and balanced Yin and Yang (Fang et al., 2012). Amounting evidence of animal experiments have shown that acupuncture at the points located in the head and the neck could improve the blood circulation system of the brain, dilate brain blood vessel, improve the microcirculation, ameliorate cerebral edema, and activate the repair function of cerebral neurons to promote functional recovery (Kim et al., 2011; Chuang et al., 2007; Liu et al., 2012). Quchi (LI11) is the He-Sea point of the large intestine channel of Hand-Yangming. Electroacupuncture at Quchi (LI11) and Zusanli (ST36) acupoints was reported to improve the ischemia-associated scores of neurological deficits, reduce cerebral infarction, alleviate inflammatory responses, promote neovascularization, inhibit neural cell 


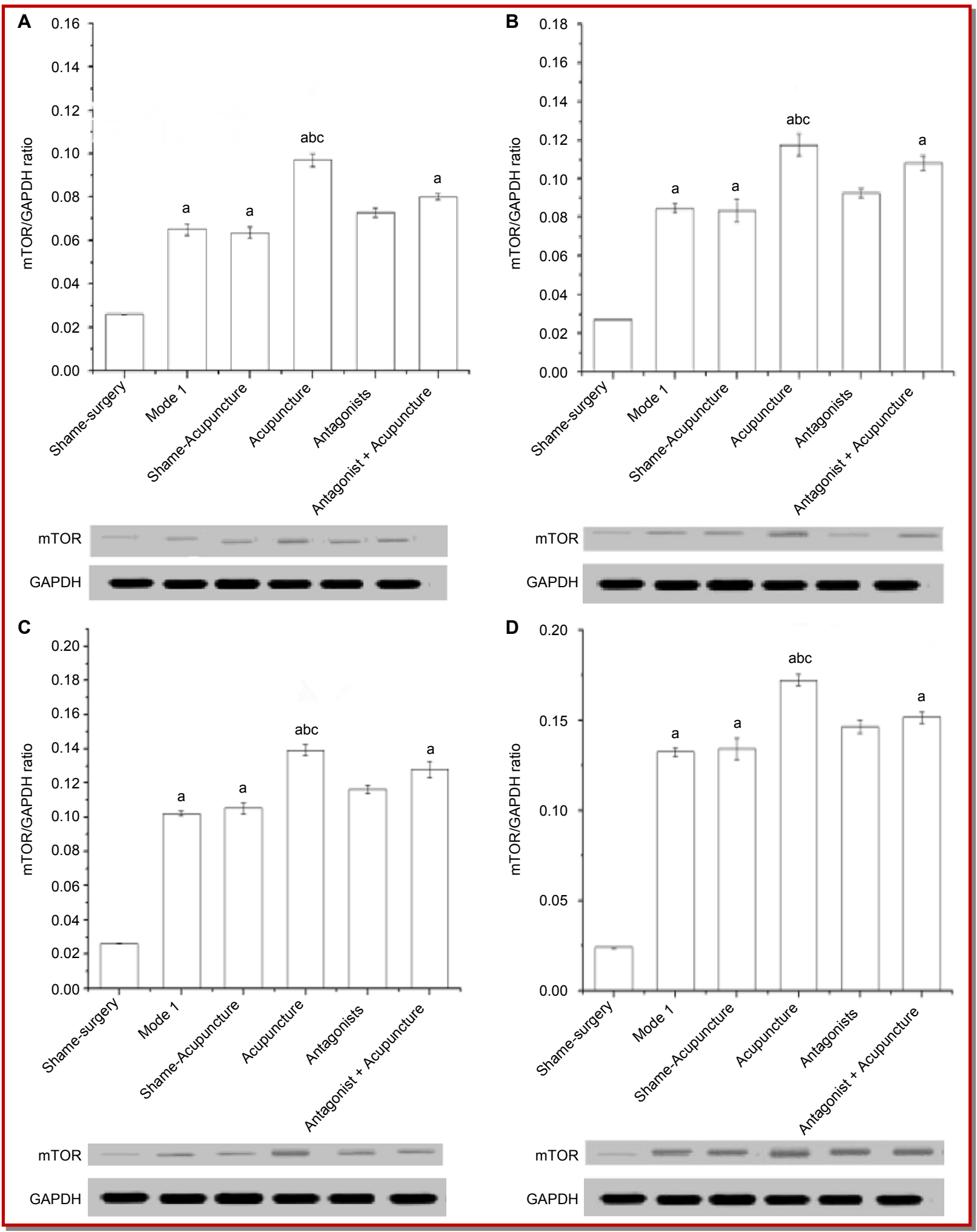

Figure 3: Effect of electroacupuncture treatment on mTOR protein expression in the injured cortex of rat with HIE (Western blot) (A)-(D) mTOR protein expression at the 1st, 3rd , 7th and 21st day respectively

Bar graphs show densitometric analysis of Western blot of mTOR protein. The densitometric quantification was normalized to the internal control GAPDH. Data are expressed as mean \pm SD $\left(n=6\right.$ rats per group, ap $<0.05$ vs the sham-surgery group; ${ }^{b} p<0.05$ vs the model group; $c p<0.05$ vs the antagonists group) 
apoptosis and promote neurological functional recovery in a focal cerebral ischemia-reperfusion injured rat model (Lan et al., 2013; Hong et al., 2013; Chen et al., 2012; Zhao et al., 2010). Yongquan (KI1) is the significant Jing-Well point of the kidney channel of Foot-Shaoyin. Using in combination with Baihui (GV20), it was shown to improve energy and intelligence, clear the brain collaterals and coordinate the channels and collaterals (Xue et al., 2009; Xue et al., 2011). Acupuncture at Quchi (LI11) and Yongquan (KI1) was reported to promote functional recovery of the extremities after neuron injury (Ji et al., 2009; Zhou et al., 2009). Considering that Baihui (GV20), Dazhui (GV14), Quchi (LI1) and Yongquan (KI1) are commonly used in clinics for patient treatment and are easy to locate, we chose the combination of the above four acupoints in our experiments. HE staining results showed that electroacupuncture treatment significantly ameliorated neuronal damage caused by hypoxicischemic injury and protected structure of neurons against the degenerative alterations, suggesting that electroacupuncture at Baihui (GV20), Dazhui (GV14), Quchi (LI11) and Yongquan (KI1) exerts neuroprotective function in HIE.

The mammalian target of rapamycin (mTOR), also termed the mechanistic target of rapamycin and FK 506binding protein 12-rapamycin complex-associated protein 1 (FRAP 1), is a $289 \mathrm{kDa}$ protein serine/ threonine protein kinase that plays a critical role in protein synthesis, cytoskeletal organization, cellular differentiation, development, survival, and aging (Chong et al., 2010). A large number of studies showed that the abnormal regulation of mTOR signaling pathway is closely related to cell proliferation. MTOR played an important role not only in the physiological processes such as cell growth, protein expression, protein degeneration, signal transduction, ribosome synthesis, but also in the formation and development of brain, the occurrence of neural synapse, the formation of learning and memory (Schicknik et al., 2008). Dying neurons in HIE may experience various morphologic changes, through apoptosis, autophagy and necrosis cell death pathways (Blomgren et al., 2007). mTOR can participate in the regulation of neuronal death. When the living conditions are changed, the external stimuli such as hunger, hypoxia, and nutritional deficiency can activate autophagy in order to survive cells. However, if the level of autophagy is too high, cells will inhibit autophagy by increasing the expression of mTOR. Special residues phosphorylation of mTOR can change its activity and regulate autophagy. In recent years, electroacupuncture treatment in hypoxic ischemic brain damage has been paid more and more attention. Severe hypoxia can lead to cell death, and mTOR as a key regulator of autophagy, can inhibit the activation of autophagy.

In the present study, we investigated the expression changes of mTOR in the hypoxic-ischemic injured cerebral cortex at the 1st, 3rd, 7th and 21st day postsurgery and explored the interventing effect of electroacupuncture. Results showed that the expression of mTOR in model group increased at the 1st day after hypoxia, and the upward trend was more obvious at the 7 th day, and it was not obvious from 7 th day to 21 st day. This suggests that rats have self protection after hypoxia ischemia, but the self protective ability are effective only in a short period of time without interventions. The change of mTOR in electroacupuncture group showed a continuous upward trend, compared to model group at 1st, 3rd, 7th, and 21rd day. The differences were significant.

\section{Conclusion}

Electroacupuncture treatment protected cortical neurons against HIE-induced neuronal damage and degenerative changes in rats, which is in association with activation of mTOR. Therefore, electroacupuncture may become a potential therapeutic strategy for HIE of newborn.

\section{Ethical Issue}

All experimental procedures were performed in accordance with the Guidance Suggestions for the Care and Use of Laboratory Animals, formulated by the Ministry of Science and Technology of the People's Republic of China.

\section{Acknowledgement}

The project was supported by the Natural Science Foundation of China (NO. 81202761).

\section{References}

Bellot G, Garcia-Medina R, Gounon P, et al. Hypoxia-induced autophagy is mediated through hypoxia-inducible factor induction of BNIP3 and BNIP3L via their BH3 domains. Mol Cell Biol. 2009; 29: 2570-81.

Blagosklonny MV. Hypoxia, MTOR and autophagy: Converging on senescence or quiescence. Autophagy 2013; 9: 260-62.

Blomgren K, Leist M, Groc L, et al. Pathological apoptosis in the developing brain. Apoptosis 2007; 12: 993-1010.

Chen A, Lin Z, Lan L, et al. Electroacupuncture at the Quchi and Zusanli acupoints exerts neuroprotective role in cerebral ischemia-reperfusion injured rats via activation of the PI3K/Akt pathway. Int J Mol Med. 2012; 30: 791-96.

Chong ZZ, et al. Mammalian target of rapamycin: Hitting the bull's-eye for neurological disorders. Oxid Med Cell Longev. 2010; 3: 374-91.

Chuang CM, Hsieh CL, Li TC, et al. Acupuncture stimulation 
at Baihui acupoint reduced cerebral infarct and increased dopamine levels in chronic cerebral hypoperfusion and ischemia-reperfusion injured Sprague-Dawley rats. Am J Chin Med. 2007; 35: 779-91.

Fahling M. Cellular oxygen sensing, signalling and how to survive translational arrest in hypoxia. Acta Physiol (Oxf.). 2009; 195: 205-30.

Fang Z, Ning J, Xiong C, et al. Effects of electroacupuncture at head points on the function of cerebral motor areas in stroke patients: A PET study. Evid Based Complement Alternat Med. 2012: 902413.

Fatemi A, Wilson MA, Johnston MV. Hyopxic-ischemic encephalopathy in the term infant. Clin Perinatol. 2009; 36: 83558.

Gao J, Wang S, Wang X, et al. Electroacupuncture enhances cell proliferation and neuronal differentiation in young rat brains. Neurol Sci. 2011; 32: 369-74.

Gunn RM, Hailes HC. Insights into the PI3-K-PKB-mTOR signalling pathway from small molecules. Chem Biol. 2008; 1: 49-62.

Hong J, Wu G, Zou Y, et al. Electroacupuncture promotes neurological functional recovery via the retinoic acid signaling pathway in rats following cerebral ischemiareperfusion injury. Int J Mol Med. 2013; 31: 225-31.

Ji XQ, Zhang ZL. Observation on therapeutic effect of nuchal acupuncture and abdominal acupuncture for treatment of stroke patients with spastic hemiplegia. Zhongguo Zhen Jiu. 2009; 29: 961-65.

Kang J, Rychahou PG, Ishola TA, et al. Nmyc is a novel regulator of PI3K-mediated VEGF expression in neuroblastoma. Oncogene 2008; 27: 3999-4007.

Nakamura K, Martin KC, Jackson JK, et al. Thiele, brain derived neurotrophic factor activation of TrkB induces vascular endothelial growth factor expression via hypoxiainducible factor-1 alpha in neuroblastoma cells. Cancer Res. 2006; 66: 4249-55.

Kim MW, Chung YC, Jung HC, et al. Scalp electroacupuncture at the Baihui acupoint (DU 20) improves functional recovery in rats with cerebral ischemia. Neural Regen Res. 2011; 6: 2822-28.

Koike M, Shibata M, Tadakoshi M. Inhibition of autophagy prevents hippocampal pyramidal neuron death after hypoxic-ischemic injury. Am J Pathol. 2008; 172: 454-69.

Lai MC, Yang SN. Perinatal hypoxic-ischemic encephalopathy. J Biomed Biotechnol. 2011: 2011.

Lan L, Tao J, Chen A, et al. Electroacupuncture exerts antiinflammatory effects in cerebral ischemia-reperfusion injured rats via suppression of the TLR4/NF-kB pathway. Int J Mol Med. 2013; 31: 75-80.

Li M, Peng J, Song Y, et al. Electro-acupuncture combined with transcranial magnetic stimulation improves learning and memory function of rats with cerebral infarction by inhibiting neuron cell apoptosis. J Huazhong Univ Sci Technolog Med Sci. 2012; 32: 746-49.

Li L, Qu Y, Li J, Xiong Y, Mao M, Mu D. Relationship between HIF expression and neuronal apoptosis in neonatal rats with hypoxia-ischemia brain injury. Brain Res. 2007: 1180; 133-39.

Liu R, Xu N, Yi W, et al. Electroacupuncture effect on neurological behavior and tyrosine kinase-JAK 2 in rats with focal cerebral ischemia. J Tradit Chin Med. 2012; 32: 465-70.

MacPherson H, Hammerschlag R. Acupuncture and the emerging evidence base: Contrived controversy and rational debate. J Acupunct Meridian Stud. 2012; 5: 141-47.

Mazure NM, Pouysségur J. Hypoxia-induced autophagy: Cell death or cell survival? Curr Opin Cell Biol. 2010; 22: 177-80.

Rice JE, Vannucci RC, Brierley JB, et al. The influence of immaturity on hypoxic-ischemic brain damage in the rat. Ann Neurol. 1981; 9: 131-41.

Rudov A, Balduini W, Carloni S, Perrone S, Buonocore G, Cristina Albertini M. Involvement of miRNAs in placental alterations mediated by oxidative stress. Oxid Med Cell Longev. 2014: 1030-68.

Schicknik H, Schott BH, Budinger E, et al. Dopaminergic modulation of auditory cortex-dependent memory consolidation through mTOR. Cereb Cortex. 2008; 18: 264658 .

Wang GB, Xu NG, She SF, et al. Effect of electroacupuncture on the synaptic structure in the ischemic areas of rats with focal cerebral ischemia. Zhongguo Linchuang Kangfu. 2005; 9: 115-17.

Xue WG, Ge GL, Zhang Z, et al. Effect of electroacupuncture on the behavior and hippocampal ultrastructure in APP 695 V 717 I transgenic mice. Zhen Ci Yan Jiu. 2009; 34: 309-14.

Xue WG, Zhang Z, Xu H, et al. Effect of electroacupuncture on learning-memory ability, and Abeta and LRP1 immunoactivity in hippocampal sulcus microvessels in APP transgenic mice. Zhen Ci Yan Jiu. 2011; 36: 95-100.

Yang ZX, Yu HB, Wang L, et al. Effect of electroacupuncture on synaptic transmission in hippocampal dentate gyrus of rats with cerebral ischemic damage. Guangzhou Zhongyiyao Daxue Xuebao. 2005; 22: 118-22.

Yi W, Xu NG, Wang GB, et al. Experimental study on effects of electro-acupuncture in improving synaptic plasticity in focal cerebral ischemia rats. Zhongguo Zhongxiyi Jiehe Zazhi. 2006; 26: 710-14.

Zhao Y, Chen S, Yu W, et al. The effect of electroacupuncture on endogenous EPCs and serum cytokines in cerebral ischemia-reperfusion rat. Sheng $\mathrm{Wu}$ Yi Xue Gong Cheng Xue Za Zhi. 2010; 27: 1322-26.

Zhou W, Liu H, Wang LP, et al. Effect of alternative administration of scalp-acupuncture, body-acupuncture and abdominal-acupuncture on the upper-limb dyscinesia in patients with stroke. Zhen Ci Yan Jiu. 2009; 34: 128-31. 


\section{Your feedback about this paper}

1. Number of times you have read this paper 0

2. Quality of paper
Excellent
$\bigcirc$ Good
Moderate
Not good

3. Your comments

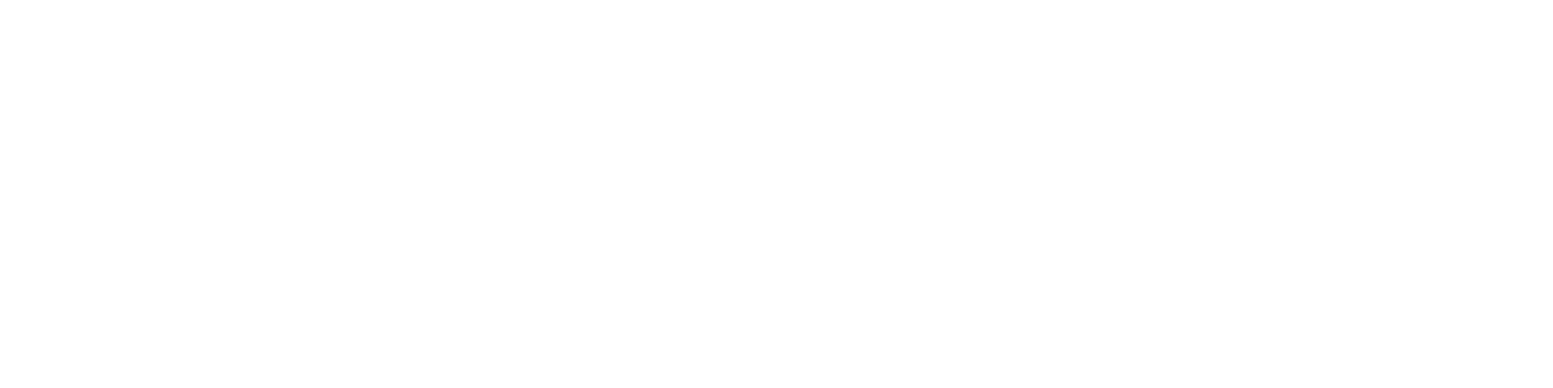

